\title{
Habilitação em acupuntura para cirurgiões-dentistas no Brasil: uma análise por estados e regiões
}

\author{
Certification in acupuncture for dentists in Brazil: \\ an analysis by states and regions \\ Capacitación en acupuntura para dentistas en Brasil: \\ un análisis por estados y regiones \\ José de Alencar FERNANDES NETO ${ }^{1}$ \\ Maria das Graças Barbosa da SILVA ${ }^{1}$ \\ Thamyres Maria Silva SIMÕES ${ }^{1}$ \\ Flávio de Almeida ANDRADE ${ }^{2}$ \\ Ana Luzia Araujo BATISTA ${ }^{3}$ \\ Maria Helena Chaves de Vasconcelos CATÃO ${ }^{4}$
}

Mestrando do Programa de Pós-Graduação em Odontologia, Departamento de Odontologia, Universidade Estadual da Paraíba, UEPB, 58429-500 Campina Grande-PB, Brasil

${ }^{2}$ Graduando em Odontologia, Departamento de Odontologia, Universidade Estadual da Paraíba, UEPB, 58429-500, Campina GrandePB, Brasil

${ }^{3}$ Doutoranda do Programa de Pós-Graduação em Odontologia, Departamento de Odontologia, Universidade Estadual da Paraíba, UEPB, 58429-500, Campina Grande - PB, Brasil

${ }^{4}$ Professora Doutora do Programa de Pós-Graduação em Odontologia, Universidade Estadual da Paraíba, UEPB, 58429-500, Campina Grande - PB, Brasil

\begin{abstract}
Resumo
Introdução: A prática da acupuntura na Odontologia ainda é pouco utilizada, apesar de ter se mostrado um método terapêutico que agrega benefícios aos tratamentos odontológicos convencionais. Objetivo: avaliar a quantidade de cirurgiões-dentistas habilitados em acupuntura e de cursos da habilitação anteriormente oferecidos, de acordo com os estados e regiões brasileiras. Material e método: Os números totais de cirurgiões-dentistas no Brasil, dos habilitados em acupuntura e as informações sobre os cursos de habilitação já ministrados no país foram coletados diretamente do site do CFO. Todos os dados utilizados nesta pesquisa são de acesso público. Resultados: São Paulo é o estado brasileiro com o maior número de habilitados em acupuntura $(n=246)$, enquanto que nove estados ainda não possuem profissional habilitado nesta prática. O Sudeste detém o maior número de habilitados em acupuntura ( $\mathrm{n}=305$ ), correspondendo a $74,4 \%$ do total encontrado em todo o Brasil $(n=410)$. Apenas quatro profissionais $(1,0 \%)$ estão localizados no Norte, região onde para cada habilitado em acupuntura existem 3.667,8 cirurgiões-dentistas $(1 / 3.667,8)$. Quanto aos cursos da habilitação oferecidos no país, todos $(n=12)$ foram realizados em dois estados: Paraná (n=8) e São Paulo. Conclusão: Conclui-se que há uma pequena quantidade de cirurgiões-dentistas habilitados e de cursos de habilitação em acupuntura em todo o Brasil, principalmente nas regiões Norte, Nordeste e CentroOeste.
\end{abstract}

Descritores: Acupuntura; Terapia por Acupuntura; Odontologia; Odontólogos; Credenciamento; Currículo.

\begin{abstract}
Introduction: The practice of acupuncture in dentistry is little used yet, although it has proved to be a therapeutic method that adds benefits to conventional dental treatments. Objective: To evaluate the number of dentists qualified in acupuncture and the qualification courses already offered, according to the Brazilian states and regions. Material and method: The total numbers of dentists in Brazil, those trained in acupuncture and the information about habilitation courses already taught in the country were collected directly from the CFO's website. All data used in this research are of public access. Results: São Paulo is the Brazilian state with the highest number of certified dentists in acupuncture ( $\mathrm{n}=246$ ), while nine states do not yet have a qualified professional in this practice. The Southeast has the highest number of qualified dentists in acupuncture $(n=305)$, corresponding to $74.4 \%$ of the total found in Brazil $(\mathrm{n}=410)$. Only four professionals $(1.0 \%)$ are located in the North, where for each qualified professional there are $3,627.8$ dentist $(1 / 3.667,8)$. About the qualification courses offered in the country, all $(n=12)$ were carried out in two states: Paraná $(n=8)$ and São Paulo. Conclusion: It is concluded that there is a small number of qualified dentists and of acupuncture qualification courses throughout Brazil, mainly in the North, Northeast and Central-West regions. Descriptors: Acupuncture; Acupuncture Therapy; Dentistry; Dentists; Credentialing; Curriculum.
\end{abstract}

\section{Resumen}

Introducción: La práctica de la acupuntura en odontología aún es poco utilizada, a pesar de que ha demostrado ser un método terapéutico que aporta beneficios a los tratamientos dentales convencionales. Objetivo: Evaluar la cantidad de dentistas cualificados en la acupuntura y el número de cursos de la capacitación, de acuerdo con los estados y regiones. Material y método: El número total de dentistas en Brasil, de los dentistas cualificados en la acupuntura y las informaciones sobre los cursos de capacitación en el país se recogieron directamente desde el sitio de lo CFO. Todos los datos utilizados en esta investigación son de acceso público. Resultados: São Paulo es el estado brasileño con el mayor número de personal cualificado en la acupuntura $(\mathrm{n}=246)$, mientras que nueve estados todavía no tienen un profesional cualificado en esta práctica. El sudeste tiene el mayor número de dentistas cualificados en la acupuntura $(n=305)$, lo que corresponde al $74,4 \%$ del total que se encuentra en Brasil $(n=410)$. Sólo cuatro profesionales $(1,0 \%)$ se encuentran en la región Norte, donde para cada cualificado en la acupuntura son 3.667,8 dentistas (1/3.667.8). Sobre los cursos de capacitación que se ofrecen en el país, todos $(n=12)$ fueron realizados en dos estados: Paraná $(n=8)$ y São Paulo. Conclusión: Se concluye que hay una pequeña cantidad de dentistas calificados y cursos de capacitación en acupuntura en Brasil, sobre todo en el Norte, Nordeste y Centro-Oeste.

Descriptores: Acupuntura; Terapia por Acupuntura; Odontología; Odontólogos; Habilitación Profesional; Curriculum. 


\section{INTRODUÇÃO}

A acupuntura é uma terapia integrante da Medicina Tradicional Chinesa (MTC) que a partir da década de 70 foi introduzida no ocidente, tendo se desenvolvido e se integrado com a medicina convencional no tratamento e na prevenção de diversos problemas e doenças, devido às suas propriedades anti-inflamatórias, ansiolíticas, miorelaxantes e ativadoras da função imunológica, propiciando melhor qualidade de vida, bem-estar e saúde ao paciente ${ }^{1-3}$.

Em 2008, através da Resolução federal CFO-82/2008, o Conselho Federal de Odontologia (CFO), reconheceu e regulamentou o uso pelo cirurgião-dentista de práticas integrativas e complementares à saúde bucal, incluindo a acupuntura ${ }^{4}$. Desde então, surgiram no país os cursos de habilitação em acupuntura para cirurgiões-dentistas, com o objetivo de habilitar o profissional nessa terapia, capacitando-o a utilizá-la em procedimentos clínicos odontológicos.

São algumas das atribuições do habilitado em acupuntura: incorporar à ciência da acupuntura como instrumento da arte de curar na prática profissional odontológica, atuar em todos os níveis de atenção à saúde, sempre sensibilizados e comprometidos com o ser humano, respeitando-o e valorizando-o, segundo os fundamentos da prática da MTC e da ciência atual e desenvolver, participar e aplicar conhecimento, que objetivem a qualificação da prática profissional com base nos pressupostos da MTC ${ }^{4}$.

Segundo Pereira et al. ${ }^{2}$ a prática da acupuntura na Odontologia ainda é pouco utilizada e estudada, apesar de ser um método terapêutico não invasivo e que agrega benefícios aos tratamentos odontológicos convencionais. Além disso, considera o paciente integralmente e busca otimizar resultados, podendo realmente ser considerada uma terapia alternativa, integradora e complementar.

Dentre as indicações da acupuntura na clínica odontológica estão: tratamento das disfunções temporomandibulares ${ }^{5-8}$, xerostomia ${ }^{9}$, controle de dores orofaciais $^{10,11}$, e parestesias ${ }^{1,12}$.

Diante disso, este trabalho teve como objetivo avaliar a quantidade de cirurgiões-dentistas habilitados em acupuntura e de cursos da habilitação anteriormente oferecidos, de acordo com os estados e regiões brasileiras.

\section{MATERIAL E MÉTODO}

Os números totais de cirurgiões-dentistas no Brasil, dos habilitados em acupuntura e as informações sobre os cursos da habilitação anteriormente ministrados no país foram coletados diretamente do site do $\mathrm{CFO}^{13}$, assim como a distribuição geográfica desses de acordo com as unidades federativas brasileiras.

$\mathrm{Na}$ pesquisa, foram considerados apenas aqueles profissionais que estavam em atividade, de acordo com o registro no Conselho. Aqueles com as situações "cancelado" ou "falecido" não foram contabilizados, bem como aqueles que se transferiram para outro estado, pois foi levada em consideração apenas a localidade atual.

Quanto aos cursos de habilitação em acupuntura, foram recolhidas as seguintes informações também no site do $\mathrm{CRO}^{13}$ : local do curso, período, carga horária e número de vagas oferecidas.

A pesquisa foi realizada no dia 10 de novembro de 2016, conseguindo-se assim o número exato de profissionais e de cursos da habilitação anteriormente oferecidos em cada estado, devidamente cadastrados no Conselho, até a data da busca. Todos os dados utilizados nesta pesquisa são de acesso público.

\section{RESULTADOS}

De acordo com a pesquisa, São Paulo é o estado brasileiro com o maior número de habilitados em acupuntura $(n=246)$, enquanto que nove estados ainda não possuem profissional habilitado nesta prática $(\mathrm{n}=0)$ : Alagoas, Amapá, Maranhão, Pará, Paraíba, Piauí, Rondônia, Sergipe e Tocantins. Quanto aos cursos da habilitação oferecidos no país, todos $(\mathrm{n}=12)$ foram realizados em dois estados: Paraná $(n=8)$ e São Paulo $(n=4)$ (Tabela 1).

Tabela 1. Distribuição do número de cursos da habilitação ministrados, de profissionais habilitados e relação habilitados/cirurgião-dentista por unidade federativa brasileira

\begin{tabular}{|c|c|c|c|c|}
\hline $\begin{array}{l}\text { Unidade } \\
\text { Federativa }\end{array}$ & $\begin{array}{l}\text { Cursos da } \\
\text { Habilitação } \\
\text { ministrados }\end{array}$ & $\begin{array}{l}\text { Habilitados } \\
\text { em } \\
\text { acupuntura }\end{array}$ & $\begin{array}{l}\text { Número de } \\
\text { Cirurgiões- } \\
\text { Dentistas }\end{array}$ & $\begin{array}{c}\text { Relação } \\
\text { Habilitados/ } \\
\text { Cirurgião- } \\
\text { Dentista }\end{array}$ \\
\hline & $\mathbf{n}$ & $\mathbf{n}$ & $\mathrm{n}$ & \\
\hline Acre & 0 & 1 & 645 & $1 / 645$ \\
\hline Alagoas & 0 & 0 & 2.710 & $0 / 2.710$ \\
\hline Amapá & 0 & 0 & 657 & $0 / 657$ \\
\hline Amazonas & 0 & 2 & 3.798 & $1 / 1.899$ \\
\hline Bahia & 0 & 2 & 11.358 & $1 / 5.679$ \\
\hline Ceará & 0 & 9 & 6.441 & $1 / 715,7$ \\
\hline Distrito Federal & 0 & 6 & 6.978 & $1 / 1.163$ \\
\hline Espírito Santo & 0 & 4 & 5.499 & $1 / 1.374,8$ \\
\hline Goiás & 0 & 8 & 9.829 & $1 / 1.228,6$ \\
\hline Maranhão & 0 & 0 & 3.728 & $0 / 3.728$ \\
\hline Mato Grosso & 0 & 2 & 4.344 & $1 / 2.172$ \\
\hline Mato Grosso do Sul & 0 & 3 & 3.845 & $1 / 1.281,7$ \\
\hline Minas Gerais & 0 & 25 & 33.328 & $1 / 1.333,1$ \\
\hline Pará & 0 & 0 & 4.916 & $0 / 4.916$ \\
\hline Paraíba & 0 & 0 & 4.190 & $0 / 4.190$ \\
\hline Paraná & 8 & 50 & 18.019 & $1 / 360,4$ \\
\hline Pernambuco & 0 & 4 & 7.753 & $1 / 1.938,6$ \\
\hline Piauí & 0 & 0 & 2.779 & $0 / 2.779$ \\
\hline Rio de Janeiro & 0 & 30 & 30.410 & $1 / 1.013,7$ \\
\hline Rio Grande do Norte & 0 & 3 & 3.562 & $1 / 1.187,3$ \\
\hline Rio Grande do Sul & 0 & 8 & 17.457 & $1 / 2.182,1$ \\
\hline Rondônia & 0 & 0 & 2.059 & $0 / 2.059$ \\
\hline Roraima & 0 & 1 & 630 & $1 / 630$ \\
\hline Santa Catarina & 0 & 6 & 11.312 & $1 / 1.885,3$ \\
\hline São Paulo & 4 & 246 & 85.192 & $1 / 346,3$ \\
\hline Sergipe & 0 & 0 & 1.900 & $0 / 1.900$ \\
\hline Tocantins & 0 & 0 & 1.966 & $0 / 1.966$ \\
\hline Total & 12 & 410 & 285.305 & $1 / 695,9$ \\
\hline
\end{tabular}

*Total de cursos de Habilitação em Acupuntura ministrados e cadastrados no CFO.

**Totalização geral dos inscritos em atividade no Brasil de acordo com o CFO.

Por regiões brasileiras, o Sudeste detém o maior número de habilitados em acupuntura ( $\mathrm{n}=305)$, correspondendo a 74,4\% do total encontrado em todo o Brasil (n=410). Apenas 4 profissionais $(1,0 \%)$ estão localizados no Norte, região onde para cada habilitado em acupuntura existem $3.667,8$ cirurgiões-dentistas $(1 / 3.667,8)$ (Tabela 2).

Tabela 2. Distribuição percentual de cursos da habilitação ministrados de profissionais habilitados e relação habilitados/cirurgião-dentista por unidade federativa brasileira

\begin{tabular}{lcccc}
\hline $\begin{array}{c}\text { Região } \\
\text { brasileira }\end{array}$ & $\begin{array}{c}\text { Cursos de } \\
\text { Habilitação } \\
\text { ministrados* }\end{array}$ & $\begin{array}{c}\text { Habilitados } \\
\mathbf{e m} \\
\text { acupuntura }\end{array}$ & $\begin{array}{c}\text { Número de } \\
\text { Cirurgiões- } \\
\text { Dentistas }\end{array}$ & $\begin{array}{c}\text { Relação } \\
\text { habilitados/cirurgião } \\
\text {-dentista }\end{array}$ \\
\hline Centro- & $\mathbf{n}(\%)$ & $\mathbf{n}(\%)$ & $\mathbf{n}(\%)$ & \\
Oeste & $0(0 \%)$ & $19(4,6 \%)$ & $24.996(8,8 \%)$ & $1 / 1.315,6$ \\
Nordeste & $0(0 \%)$ & $18(4,4 \%)$ & $44.421(15,6 \%)$ & $1 / 2.467,8$ \\
Norte & $0(0 \%)$ & $4(1,0 \%)$ & $14.671(5,1 \%)$ & $1 / 3.667,8$ \\
Sudeste & $4(33,3 \%)$ & $305(74,4 \%)$ & $154.429(54,1 \%)$ & $1 / 506,3$ \\
Sul & $8(66,7 \%)$ & $64(15,6 \%)$ & $46.788(16,4 \%)$ & $1 / 731,1$ \\
\hline Total & $\mathbf{1 2 ( 1 0 0 \% )}$ & $\mathbf{4 1 0 ( 1 0 0 \% )}$ & $\mathbf{2 8 5 . 3 0 5 ( 1 0 0 \% )}$ & $\mathbf{1 / 6 9 5 , 9}$ \\
\hline
\end{tabular}

*Total de cursos de Habilitação em Acupuntura ministrados e cadastrados no CFO

**Totalização geral dos inscritos em atividade no Brasil de acordo com o CFO.

Constatou-se que o primeiro curso de habilitação em acupuntura foi iniciado em 2008, no estado de São Paulo. Com relação às cargas horárias dos cursos, verificou-se que a mínima foi de 350 e a máxima, 610 horas (Tabela 3). 
Tabela 3. Características dos cursos de habilitação em acupuntura de acordo com os dados do CFO

\begin{tabular}{|c|c|c|c|c|}
\hline \multicolumn{5}{|c|}{ Paraná } \\
\hline Curso & Local & Período & $\begin{array}{l}\text { Carga } \\
\text { Horária }\end{array}$ & $\begin{array}{l}\text { Número de } \\
\text { vagas } \\
\text { oferecidas }\end{array}$ \\
\hline Curso 1 & Curitiba & $2011-2012$ & 350 & 16 \\
\hline Curso 2 & Curitiba & $2012-2014$ & 350 & 12 \\
\hline Curso 3 & Curitiba & $2014-2016$ & 480 & 12 \\
\hline Curso 4 & Curitiba & $2011-2013$ & 360 & 20 \\
\hline Curso 5 & Curitiba & $2010-2011$ & 610 & 12 \\
\hline Curso 6 & Curitiba & $2013-2014$ & 360 & 30 \\
\hline Curso 7 & Curitiba & $2013-2014$ & 360 & 30 \\
\hline Curso 8 & Curitiba & $2015-2017$ & 360 & 30 \\
\hline \multicolumn{5}{|c|}{ São Paulo } \\
\hline Curso & Local & Data & $\begin{array}{c}\text { Carga } \\
\text { Horária }\end{array}$ & $\begin{array}{l}\text { Número de } \\
\text { vagas } \\
\text { oferecidas }\end{array}$ \\
\hline Curso 1 & Campinas & $2010-2012$ & 384 & 20 \\
\hline Curso 2 & São José do Rio Preto & $2008-2010$ & 350 & 12 \\
\hline Curso 3 & São José do Rio Preto & $2011-2012$ & 350 & 12 \\
\hline Curso 4 & Ribeirão Preto & $2013-2015$ & 400 & 22 \\
\hline
\end{tabular}

\section{DISCUSSÃO}

A acupuntura, quando seguidas as diretrizes recomendadas e praticada por profissionais capacitados, tende a trazer benefícios tanto ao paciente quanto para o cirurgião-dentista, como por exemplo: otimização de tempo no consultório, efeitos analgésicos, anti-inflamatórios e menor prescrição e administração de medicamentos sistêmicos e anestésicos ${ }^{14,15}$.

Chama a atenção o fato de apenas dois estados brasileiros terem oferecido cursos de habilitação em acupuntura e apesar disso, ter profissionais habilitados nas cinco regiões do Brasil. Uma das explicações para esse fato, pode ser devido os cirurgiões-dentistas terem realizado o curso de habilitação em um dos dois estados e retornaram para as cidades onde atuam profissionalmente.

A Resolução CFO-82/2008 ${ }^{4}$ também deixa claro que o profissional que em até 180 dias da data da publicação da mesma, comprovou utilizar acupuntura, há cinco anos dentro dos últimos dez anos, pôde requerer habilitação, após devida análise pelo Conselho. Isso pode justificar tanto o número de habilitados em acupuntura $(n=410)$ ser maior que a quantidade de vagas totais oferecidas pelos cursos da habilitação $(n=228)$, quanto a presença de habilitados em regiões que nunca ofereceram os cursos.

Uma justificativa para o reduzido número de profissionais habilitados pode ser devido ao também pequeno número de cursos oferecidos, inclusive centralizados apenas em dois estados das regiões sudeste e sul, ficando descobertas Norte, Nordeste e Centro-Oeste do Brasil.

Rui et al. $^{3}$ ao verificarem o conhecimento e o interesse em acupuntura de 400 pacientes que utilizaram o Centro de Especialidades Odontológicas (CEO) do Sistema Único de Saúde (SUS) nos municípios de Rio Claro e Cerquilho, em São Paulo, observaram que a maioria dos usuários em ambos os municípios conhecia a acupuntura e tinha interesse na oferta desta técnica pelo SUS, apesar de poucos terem relatado ter acesso a este tratamento. Apenas 1 $(0,5 \%)$ paciente em cada município declarou ter feito tratamento com acupuntura relacionado à área odontológica (disfunções temporomandibulares).

Para Vasconcelos et al. ${ }^{11}$, apesar da acupuntura ser uma terapia milenar, ainda tem um campo vasto a ser explorado, principalmente no que se diz respeito à sua aplicabilidade na odontologia. Os cirurgiões-dentistas devem fazer cursos para se habilitar à realização desse tipo de tratamento nos seus consultórios, oferecendo mais um método terapêutico não invasivo e de eficácia reconhecida para seus pacientes.

Deve-se levar em consideração também que alguns profissionais possam ter realizado o curso e não terem cadastrado a habilitação no CFO. Além disso, nos estados que apresentam profissionais habilitados, estes podem estar centralizados em apenas cidades de médio ou grande porte gerando uma distribuição desigual.

Todos os cursos da habilitação respeitaram a carga horária mínima de 350 horas exigida pelo CFO através da Resolução CFO-82/2008 ${ }^{4}$.

\section{CONCLUSÃO}

Com este estudo, conclui-se que há uma pequena quantidade de cirurgiões-dentistas habilitados em acupuntura em todo o Brasil, principalmente nos estados das regiões Norte, Nordeste e Centro-Oeste. O aumento do número de cursos de habilitação em acupuntura, principalmente nas regiões que nunca ofereceram, poderia propiciar um crescimento no número de profissionais habilitados nessa técnica, de forma que a acupuntura seja sempre utilizada de forma segura e eficaz, trazendo benefícios aos tratamentos odontológicos.

\section{REFERÊNCIAS}

1. Florian MR, Rando-Meirelles MPM, Sousa MLR. Uso da acupuntura em um caso de parestesia dos nervos alveolar inferior e lingual. Rev Assoc Paul Cir Dent. 2012; 66(4):312-5

2. Pereira MSS, Silva BO, Santos FR. Acupuntura: terapia alternativa, integrativa e complementar na Odontologia. R CROMG. 2015; 16(1):19-26.

3. Rui AAO, Florian MR, Grillo CM, Meirelles MPMR, Sousa MLR. Avaliação do conhecimento e interesse em tratamento com Acupuntura na Odontologia pelos usuários do Sistema Único de Saúde dos municípios de Rio Claro e Cerquilho-SP. J Health Sci Inst. 2013; 31(1):24-6.

4. CFO. Conselho Federal de Odontologia. Resolução $n^{\circ}$ 82 de 25 de setembro de 2008. DOU 190 de 01/10/2008. p.105-11.

5. Borin GS, Corrêa EC, Silva AMT, Milanesi JM. Acupuntura como recurso terapêutico na dor e na gravidade da desordem temporomandibular. Fisioter Pesqui. 2011; 18(3):217-22.

6. Borin GS, Corrêa ECR, Silva AMT, Milanesi JM. Avaliação eletromiográfica dos músculos da mastigação de indivíduos com desordem temporomandibular submetidos a acupuntura. Rev Soc Bras Fonoaudiol. 2012; 17(1):1-8.

7. Alves Rezende MCR, Sant'Anna CBM, Ávila de Aguiar SMHC, Bertoz APM, Hall KB, Dyonisio ALDB et al. Temporomandibular Disorders in Females: Acupuncture Compared to Occlusal Splint. Arch Health Invest. 2013; 2(3):8-14.

8. Sousa MLR, Mashuda CS, Sato JE, Siqueira JTT. Effects of acupuncture in adults with temporomandibular disorders. Rev Dor. São Paulo. 2014; 15(2):87-90.

9. Saito LT, Silvério-Lopes S, Pontes SRB, Suliano LC Acupuntura sistêmica no tratamento de xerostomia decorrente do uso de anti-hipertensivos: estudo de casos. Rev Bras Terap e Saúde. 2012; 3(1):9-13. 
10. Boleta-Ceranto DCF, Alves T, Alende FL. O efeito da acupuntura no controle da dor na odontologia. Arq Ciênc Saúde Unipar. 2008; 12(2):143-8.

11. Vasconcelos FHP, Catão MHCV, Pereira FG, Janoca MIG, Abrantes Segundo JHG, Florentino VGB. Acupuntura em odontologia: uma revisão de literatura. Rev Bras Ciên Saúde. 2011; 9(28):38-42.

12. Castro ALF, Miranda FP, Pedras RBN, Noronha VRAS. Tratamento da parestesia do nervo alveolar inferior e lingual no pós operatório de $3^{\circ}$ molar: revisão de literatura. R CROMG. 2015; 16(2):34-42.

13. CFO: Conselho Federal de Odontologia. [Internet]. Citado em 2016 nov 10. Disponível em: http://cfo.org.br/

14. Vianna RS, Souza AG, Silva BC, Berlinck TÁ, Dias KRHC. A Acupuntura e sua aplicação na Odontologia UFES Rev Odontol. 2008; 10(4):48-52.

15. Rui A, Meirelles MPMR, Sousa MLR. Relato de caso sobre o uso da acupuntura no tratamento da dor orofacial. Arq Ciênc Saúde UNIPAR. 2011; 15(3):287-90

\section{CONFLITO DE INTERESSES}

Os autores declaram não haver conflitos de interesse.

\section{AUTOR PARA CORRESPONDÊNCIA}

Maria Helena Chaves de Vasconcelos Catão

mhelenact@zipmail.com.br

Submetido em 23/02/2017 Aceito em 03/04/2017 